\title{
Measuring the Cost of Economic Fluctuations with Preferences that Rationalize the Equity Premium
}

\author{
Angelo Melino* \\ Department of Economics \\ University of Toronto \\ 150 St. George St. \\ Toronto, Canada \\ M5S 3G7 \\ angelo.melino@utoronto.ca
}

September 17, 2006

\begin{abstract}
Lucas (2003) argues that the potential welfare gains from stabilizing the business cycle are small. In fact, he shows that the benefits of eliminating all economic fluctuations are small, both in an absolute sense and when compared to the potential gains from other reforms. His estimates are obtained using standard preferences. In this paper, I show that a model consistent with observed data on asset returns leads to very different conclusions. Calibrating preferences to observed asset market data raises the estimated welfare gains from completely eliminating aggregate fluctuations by approximately two orders of magnitude. Most of the gains, however, come from the elimination of low frequency contributions.
\end{abstract}

*Thanks to Alan Yang and the participants from seminars at Toronto, Siena, Rimini, and the EUI for their comments. 


\section{Introduction}

In his Presidential Address to the American Economic Association, Lucas (2003) argues that macroeconomists' efforts to control the business cycle have succeeded for all practical purposes. While not declaring the American business cycle dead, he points out that it has been much tamed since the Great Depression. Lucas claims the welfare gains that can be achieved from further reductions in business cycle fluctuations, due to additional improvements in short-run demand management, would be extremely modest. On the other hand, he lists a variety of feasible changes in long run monetary and fiscal policy that would improve the economy's average performance, or even its growth rate, and would yield substantial improvements in welfare. Lucas argues that the focus of macroeconomists' research, along with their efforts to educate the public so as to improve economic outcomes, should shift away from the business cycle toward other priorities.

To make his point, Lucas (2003) estimates the welfare gains from eliminating all fluctuations around trend in a simple endowment economy calibrated to match the aggregate consumption fluctuations of the post-War U.S. economy. This device allows him to avoid two difficult questions: which fluctuations should policy attempt to offset and which of these can it? In the endowment economy, all fluctuations in consumption around its growth rate are welfare reducing. However, some fluctuations in the U.S. economy reflect real shocks which no welfare maximizing government should offset, even if it could. So Lucas argues that his estimate of the welfare gains from eliminating completely the business cycle provides a credible upper bound on the welfare gains that are available in practice. Lucas' estimates of this upper bound are tiny: Consumers would be unwilling to give up more than a $0.2 \%$ reduction in their level of consumption each

period to eliminate economic fluctuations. By comparison, he reports that reducing inflation from $10 \%$ per annum to $0 \%$ would yield twenty times the welfare gain from eliminating the business cycle.

Whatever the merits of Lucas' thesis about where to best employ macreconomists' research efforts, his argument is deeply flawed. If we follow his approach and apply it not just to the post-War period, but to a longer sample that extends back to the dawn of the twentieth century, we continue to obtain very similar estimates for the welfare gains. Taken literally, this says that the efforts to control the business cycle were never worthwhile. The welfare gains estimated using Lucas' framework from eliminating the aggregate economic fluctuations observed in the US during the twentieth century, including a one in eight chance of a decade like the 1930s, are dwarfed by the gains from reducing inflation from $10 \%$ to $0 \%$ ! Such a nonsensical conclusion should make all macreconomists very uncomfortable, and it casts serious doubt on the 
evidence that Lucas presents. To be taken seriously, any estimate of the welfare gains from stabilization policy should assign a high value to the elimination of an economic fluctuation of the order of the Great Depression.

One of the serious problems with the calculations in Lucas (2003), which he notes in his discussion, is that the simple model he uses is inconsistent with observed asset prices. This is an important failure because financial markets tell us that the cost of bearing aggregate risk is high. In this paper, I re-examine Lucas' calculations about the welfare costs of the business cycle with one important difference: I use preferences calibrated to match observed asset prices. I show that doing so leads to estimates of the welfare gains from eliminating economic fluctuations completely that are two orders of magnitude larger than those reported by Lucas (2003). Having discarded Lucas' debating point about the low payoff to eliminating all economic fluctuations, we still are faced with the hard questions of what sort of fluctuation can policy offset and what are the potential gains from improvement in current policy. I provide some evidence on the latter. In a decomposition of the gains from eliminating economic fluctuations, I find that the welfare gains from further 'fine-tuning' the economy to smooth out high-frequency fluctuations are very small. To the extent that it is possible, the large potential gains from further improvements in stabilization policy will come from reducing fluctuations in a way that diminishes the medium and long-horizon uncertainty about aggregate consumption. Macroeconomists realize that there is no clean way to decompose the effects of policy into business cycle and longer horizon frequencies; the same shocks feed into both the cycle and lower frequency fluctuations. So it is not obvious how to think about the welfare consequences of stabilization policy based on a statistical decomposition of the uncertainty into different frequencies. Quite frankly, we have not thought very hard about how stabilization policy may effect uncertainty about the medium-run and the long-run growth rate, as opposed to the growth rate itself. Thinking a bit harder about Lucas' thesis leads to an important insight: Understanding better the sources of medium and long-horizon uncertainty and if such uncertainty can be reduced by government actions should be a topic of high priority for macroeconomists.

The potential welfare gains from improvements in either the level or growth rate of aggregate economic activity are unquestionably large and important. But this is no reason for macroeconomists to narrow their range of interest and ignore potential improvements in stabilization policy, particularly those policies that reduce low-frequency uncertainty. Despite the importance of policies that promote long-run economic performance, as measured by the level and growth rate of aggregate economic activity, there is still plenty of room in macroeconomics for those who are interested in other things, including studying and taming economic fluctuations.

This paper is not the first to point out the importance of preferences for assessing 
the costs of economic fluctuations. Tallarini (2000) obtains a similar estimate to mine of the welfare gains from eliminating all fluctuations, using Epstein-Zin preferences with a coefficient of relative risk aversion of about 100. Tallarini's model matches quantity behaviour and the equity premium nicely, but it is inconsistent with other important moments of asset returns. My model matches both quantities and the first two moments of asset returns, so my estimate should be held with more confidence. The emphasis on exploiting asset returns to estimate the welfare costs of the business cycle was made earlier by Alvarez and Jermann (2004), who also provide similar estimates on the upper bounds to eliminating different frequencies of economic fluctuations. An important difference between my paper and theirs is that I use the Mehra-Prescott endowment economy model for consumption growth in my calculations rather than project observed consumption fluctuations on observed asset prices. This simplification allows me to span the endowment process for consumption with just two assets rather than projecting onto the very large number of assets used by Alvarez and Jermann (2004). As a consequence, I can calculate explicitly the stochastic discount factor for my model economy, which makes it very easy to describe and understand what is going on in my calculations. In addition, by using preferences consistent with market data, I am able to calculate the welfare gains directly, rather than just an upper bound.

In Section 2, I review the welfare estimates of Lucas (2003) using CES/CRRA preferences. Section 3 introduces the consumption endowment process from Mehra and Prescott (1985) and shows that Lucas' results are unaffected for all practical purposes if we use the Mehra-Prescott process rather than the endowment process in Lucas (2003). Section 4 reproduces the approach pioneered in Alvarez and Jermann (2004) and provides an upper bound on the welfare gains from eliminating completely the business cycle by calculating the cost of the constant growth path in the economy with the Mehra-Prescott endowment process. To do so, I follow Melino and Yang (2003) and solve for the stochastic discount factor process for that economy from the first two moments of the risk free rate and the return on equity. I use the same procedure to estimate an upper bound on the welfare gains from smoothing the path of aggregate consumption in a way that attenuates high frequency contributions. Section 5 uses the state-dependent preferences described in Melino and Yang (2003), which reduce to standard CES preferences for deterministic constant growth consumption paths, to provide a point estimate of the welfare gains from eliminating the business cycle completely. Section 6 concludes. 


\section{Framework for Welfare Analysis and Lucas' Estimate}

Suppose we want to compare the benefits of two policies, A and B. Let $U\left(C_{A}\right)$ and $U\left(C_{B}\right)$ denote the utility level the agent gets from the consumption levels that are associated with policies A and B, respectively. Suppose the agent prefers policy B: $U\left(C_{A}\right)<U\left(C_{B}\right)$. Lucas (2003) recommends measuring the welfare gains associated with moving from policy A to policy B by the value $\lambda>0$ that solves

$$
U\left((1+\lambda) C_{A}\right)=U\left(C_{B}\right)
$$

Notice that $\lambda$ gives the percentage by which all consumption goods under policy A, in all states and all periods, must be increased to make the agent indifferent with policy B.

Lucas (2003) reports that a number of feasible policies exist that generate values $\lambda \in[1 \%, 4 \%]$ for the U.S. For example, Lucas calculates that reducing the annual inflation rate from $10 \%$ to $0 \%$ would yield a welfare gain equal to a permanent $1 \%$ increase in consumption. He also reports that eliminating distorting capital income taxes (using other taxes to support an unchanged rate of government spending) would imply an increase of consumption along the balanced growth path of 7.5 to 15 percent. Taking into account transition costs generates for such a policy yields a value of $\lambda$ in the range of $2 \%-4 \%$. For some countries with much worse macroeconomic policies than the U.S., the gains from better policies are even larger. Lucas estimates that the welfare gain from eliminating a 200 percent annual inflation rate-a number not out of the range of experience for several South American economies-is about $\lambda=7 \%$. He also cites Prescott (2002) who estimates that the steady state welfare gain to French households of adopting US taxes on capital and consumption is $\lambda=20 \%$.

In his paper, Lucas (2003) estimates that the welfare gains from eliminating the business cycle completely are much smaller than those available from the policy changes described in the previous paragraph. Lucas (2003) posits an endowment economy populated by a representative agent with CRRA preferences. He then calculates the welfare gain from eliminating completely fluctuations in the endowment process and presents it as an upper bound of the welfare gains that could be achieved from better macroeconomic policy geared toward elimating the business cycle.

Lucas posits an endowment process for the consumption stream given by

$$
c_{t}=A e^{\mu t} e^{-\sigma^{2} / 2} \varepsilon_{t}
$$


where $\ln \left(\varepsilon_{t}\right) \sim N I D\left(0, \sigma^{2}\right)$, so $E\left(e^{-\sigma^{2} / 2} \varepsilon_{t}\right)=1$ and $E_{t-1}\left(c_{t}\right)=A e^{\mu t}$. Preferences over consumption paths are given by

$$
E_{0}\left(\sum_{t=0}^{\infty} \beta^{t} \frac{c_{t}^{1-\gamma}}{1-\gamma}\right)
$$

So the welfare gains from eliminating the business cycle completely are computed by finding the value $\lambda$ that solves

$$
E_{0}\left(\sum_{t=0}^{\infty} \beta^{t} \frac{\left((1+\lambda) c_{t}\right)^{1-\gamma}}{1-\gamma}\right)=\sum_{t=0}^{\infty} \beta^{t} \frac{\left(A e^{\mu t}\right)^{1-\gamma}}{1-\gamma}
$$

Using US annual data from 1947-2001, Lucas (2003) estimates $\sigma=.032$. Setting $\gamma=1$ yields the estimate $\lambda=.05 \%$. Increasing the coefficient of relative risk aversion to $\gamma=4$ yields the estimate $\lambda=.2 \%$

I will deviate from the Lucas (2003) calculations in two ways. The most important deviation is that I will consider preferences that are consistent with asset prices. Melino and Yang (2003) show how to construct the latter for an economy with a Mehra-Prescott endowment process, so I choose to work it rather than the process used by Lucas (2003). Another good reason for the switch is that the Lucas' endowment process given by (2.2) does not display uncertainty that is growing with the forecast horizon, a feature that turns out to be very important. I will review the Mehra-Prescott endowment process in the next section. I will then show that it leads to quantitatively similar estimates of the welfare gains of eliminating the business cycle as those reported by Lucas (2003) if we maintain CRRA preferences.

\section{The Mehra Prescott Endowment Process}

Consider an endowment economy in which consumption growth $g_{t} \equiv c_{t} / c_{t-1}$ follows a Markov process that takes on two values. Assume both points of support for consumption growth are equally likely and choose $\left(g_{l}, g_{h}\right)$ to match the first two moments of consumption growth observed using about a century span (1898-1978) of US historical data: $E(g)=1.018$ and $\sigma(g)=0.036$. This involves solving the equation system:

$$
\begin{aligned}
0.5 g_{l}+0.5 g_{h} & =E(g), \\
0.5\left(g_{l}-E(g)\right)^{2}+0.5\left(g_{h}-E(g)\right)^{2} & =s^{2}(g),
\end{aligned}
$$


Because the first equation is linear in the unknowns $\left(g_{l}, g_{h}\right)$, and the second is quadratic, there are two solutions. Under the obvious requirement $g_{l}<g_{h}$, we obtain

$$
\left[\begin{array}{l}
g_{l} \\
g_{h}
\end{array}\right]=\left[\begin{array}{l}
0.982 \\
1.054
\end{array}\right]
$$

These parameters for the endowment process and its points of support are those introduced by Mehra and Prescott (1985), and subsequently used by Weil (1989), Epstein and Zin (1990), and Epstein and Melino (1995), among others. Let $\pi_{i j}$ denote the probability of going from growth state $i$ to growth state $j$. Mehra and Prescott (1985) assume the transition probability matrix for consumption growth is symmetric and estimate that the probability of staying in the same state is 0.43 , that is,

$$
\Pi=\left[\begin{array}{cc}
\pi_{l l} & \pi_{l h} \\
\pi_{h l} & \pi_{h h}
\end{array}\right]=\left[\begin{array}{ll}
0.43 & 0.57 \\
0.57 & 0.43
\end{array}\right]
$$

The Mehra-Prescott endowment process differs from (2.2) used by Lucas (2003) in a number of ways. The Mehra-Prescott process displays a stochastic trend in consumption rather than a deterministic trend so uncertainty grows with the horizon, it generates a conditional distribution of consumption tomorrow that varies with the current value, and the innovations are not Gaussian. Also, the Mehra-Prescott calibration is based on a sample that includes the Great Depression, so it is a more volatile endowment process. Nonetheless, if we follow Lucas' argument we reach almost identical conclusions. Setting $\gamma=4$ and solving for $\lambda$ as in (2.2) yields the value $\lambda=.34 \%$. Although about two-thirds larger than the estimates reported by Lucas (2003), it does not change his fundamental conclusion that the welfare gains from eliminating the business cycle completely are small both in an absolute sense and when compared to the welfare gains available from other feasible policies.

Because my calculations are calibrated to an economy that experiences an outcome such as the Great Depression about once every century, such a small estimate for the welfare gains of eliminating business cycles raises serious concerns about the procedure used by Lucas (2003). Lucas' thesis is that the welfare gains from further reductions in business cycle fluctuations are small, at least from the perspective of the US economy at the beginning of the 21st century. Lucas did not suggest that eliminating outcomes such as the Great Depression would yield trivial welfare gains. But the model and framework which he uses to support his argument leads to the nonsensical conclusion that taming the American business cycle was not worth much. To make the point more starkly, imagine if Lucas had written his paper in 1950, calibrating to the experience of 
US business cycles from the first half of the twentieth centurey. Using data from 19001950 leads to an estimate of the volatility of consumption growth, and therefore the welfare gains from eliminating all fluctuations in consumption, that is only about $2 / 3$ larger than the estimates from 1951-2000. Because 5/3 times a tiny number is still a tiny number, something seems very wrong with the assertion that Lucas' procedure leads to a conservative upper bound on the welfare gains that can be obtained from reductions in business cycle volatility. Any procedure that is used to estimate the welfare gains from stabilization policy that does not assign a large welfare gain to avoiding debacles such as the Great Depression cannot be taken seriously. I will argue below that the problem comes from using preferences that are inconsistent with the required rewards for bearing risk that economic agents reveal through observed asset prices.

\section{Asset Prices, SDF, and Upper Bound}

In this section, I follow Melino and Yang (2003) and use the Mehra-Prescott endowment process and data on the first two moments of asset returns to solve for the equilibrium asset price process and the stochastic discount factor that prices contingent claims in such an economy. I will then use this stochastic discount factor to provide an upper bound on the gains from eliminating consumption fluctuations that mimics both the approach and the results in Alvarez and Jermann (2004).

Mehra and Prescott (1985) estimate the historical average return and standard deviation of equity to be $E(M)=1.07$ and $\sigma(M)=0.165$, and estimate the historical average return and standard deviation of $T$-bills to be $E\left(r_{f}\right)=1.008$ and $\sigma\left(r_{f}\right)=0.056$.

Melino and Yang (2003) point out that if we assume consumption growth is a sufficient statistic for asset returns, then we can use the first two moments of asset returns to solve for the points of support for the risk free rate and equity processes in the Mehra-Prescott economy ${ }^{1}$. They restrict attention to economies with stationary asset returns and a state vector given by $S_{t}=\left(s_{t}, c_{t}\right)$, where $c_{t}$ denotes the economy's endowment of consumption at time $\mathrm{t}$ and $s_{t}$ is an exogenous process that determines the evolution of endowment consumption growth $g_{t}$. Stationarity of the risk free process and the assumption that behaviour doesn't add any additional endogenous state variables implies that the risk free process is measurable with respect to $s_{t}$. This means

\footnotetext{
${ }^{1}$ Essentially the same idea appeared earlier in Epstein and Melino (1995), but they generated a unique solution by assuming that the price-earnings ratio was procyclical. Melino and Yang (2003) improved on the result by showing that the same unique solution could be obtained simply by imposing no arbitrage.
} 
that, just as with consumption growth, the risk-free rate process can take on only two values, and fitting the first two moments exactly leads to a quadratic equation with two solutions. In order for equity returns to be stationary, the price-earnings ${ }^{2}$ ratio at time $t, P_{t}$, must also be expressable as a function of $s_{t}$; then the first two moments of equity returns can be used to solve for the two values that the price-earnings ratio can realize in this economy. Again, this leads to a quadratic equation. At first glance it looks like there are four solutions for the points of support of the risk-free rate and price-earnings process that will match the first two moments of the asset returns. However, among these four 'solutions', three imply arbitrage opportunities and can be discarded. Therefore, in this simple Mehra-Prescott environment, the asset price process has transition matrix given by $(3.3)$ and points of support given by $\left(P_{l}, P_{h}\right)=(23.467,27.839)$ and $\left(r_{l}, r_{h}\right)=(1.064,0.952)$. Melino and Yang $(2003)$ show that this asset price process generates very sensible behaviour for the Sharpe ratio, the predictability of stock returns, etc. They also point out that the risk-free bond and equity span the risk associated with the state vector $S_{t}$. So the price of all other assets can be determined in the Mehra-Prescott environment from the price-earnings and risk-free rate processes. A particularly convenient way to summarize this information is through the economy's stochastic discount factor.

Hansen and Richard (1987) show that in the absence of arbitrage opportunities, there exists a (positive) stochastic discount factor $Q_{t, t+1}$ that allows us to price assets via the fundamental equation

$$
E_{t}\left(Q_{t, t+1} R_{t, t+1}^{m}\right)=1
$$

where $E_{t}$ denotes the conditional expectation operator, and $R_{t, t+1}^{m}$ denotes the return to holding asset $m$ from time $t$ to $t+1$.

In the simple Mehra-Prescott economy, we have two states and two assets (equity and the risk-free bond), so the stochastic discount factor process is uniquely determined from the asset return processes derived in the previous section. Let $Q_{i j}$ denote the discount factor that is applied in state $i$ to payoffs next period in state $j$. Using the results above ${ }^{3}$ and eq(4.1), we can compute the $2 \mathrm{x} 2$ matrix of discount factors for this economy:

\begin{tabular}{l|ll}
$Q$ & $l$ & $h$ \\
\hline$l$ & 1.862 & 0.244 \\
$h$ & 1.127 & 0.949
\end{tabular}

\footnotetext{
${ }^{2}$ Earnings and dividends are equal in the Lucas endowment economy, so the price-earnings ratio is also the price-dividend ratio. The return on the market can be written as $M_{t+1}=\left(P_{t+1}+1\right) / P_{t} * g_{t+1}$ where $P$ is the price-dividend ratio.

${ }^{3}$ Recall that the return to equity realized if we go from state $i$ at time $t$ to state $j$ at time $t+1$ is given by $M_{i j}=\left(P_{j}+1\right) / P_{i} * g_{j}$ and the risk-free rate in state $i$ is $r_{i}$.
} 
As a check on the reasonableness of the required $Q$ process given by (4.2), and indirectly on the reasonableness of using the two-state process underlying the MehraPrescott economy as an approximation, it is useful to point out that its mean and standard deviation, $E(Q)=.995$ and $\sigma(Q)=.573$, satisfy the bounds computed by Hansen and Jaganathan (1991) (see their Figure 1). The first moment of $Q$ varies somewhat across states, but most of its variation comes from the high conditional variance of $Q$ in the low growth state. A further check on the reasonableness of the $Q$ process can be obtained by computing the predicted return processes for various other assets. Yang (2001) shows that this $Q$ yields an average real yield on 10 year discount bonds of 3.5\%; the conditional yields are $4.3 \%$ in the low growth state and $2.7 \%$ in the high growth state. We stress that these predictions, as well as the results in the previous section on the returns to holding the risk-free asset and equity, follow from a fairly small set of inputs: a) the two-state Markov process for consumption growth; b) the estimated first and second moments of the returns to the risk-free asset and equity; c) the assumption that consumption growth is a sufficient statistic for the risk-free rate and price-dividend ratio in each period; and d) no arbitrage.

Once we have obtained the stochastic discount factor process given by (4.2), we can use it to compute an upper bound on the welfare gains from eliminating the business cycle. How much would the representative agent be willing to pay at time t to replace the Mehra-Prescott endowment process with one where consumption grew permanently at its expected rate $\bar{g}=1.018$ ?

Alvarez and Jermann (2004) first pointed out that we can use asset prices to construct an upper bound on the value $1+\lambda$, where $\lambda$ measures the welfare gains from replacing the Mehra-Prescott endowment sequence with one that grows deterministically at the same expected rate, at least if preferences are homothetic. The argument is easy to see from Figure 1. Let $C_{A}$ denote the initial endowment consumption process $\left(c_{t}, \widetilde{c}_{t+1}, \widetilde{c}_{t+2}, \ldots\right)$ and $P_{A}$ its price. In equilibrium, $P_{A}$ is determined by the slope of the tangent line to the indifference curve at $C_{A}$. With homothetic preferences, the slope of the tangent line to the indifference curve is constant along every ray from the origin, so we must have $P_{A}(1+\lambda) C_{A} \leq P_{A} C_{B}$ for every point $C_{B}$ that lies on the same indifference curve as $(1+\lambda) C_{A}$.

Because equity is interpreted to be a claim to the consumption sequence in a Lucas endowment economy, the analog of $P_{A} C_{A}$ in our setting is $\left(1+P_{t}\right) c_{t}$, which is the cum dividend price of equity. Let $P_{t}^{d} c_{t}$ denote the price at time t of a claim to the stream $\left(\bar{g} c_{t}, \bar{g}^{2} c_{t}, ..\right)$ of consumption from time $t+1$ to $\infty$ that grows deterministically at the 
rate $\bar{g}$. Notice that $P_{t}^{d}$ is the time t price of a real consol bond with coupons $\left(\bar{g}, \bar{g}^{2}, ..\right)$. The analog of $P_{A} C_{B}$ in our setting is $\left(1+P_{t}^{d}\right) c_{t}$, so if preferences are homothetic we must have $\left(1+\lambda_{t}\right) \leq\left(1+P_{t}^{d}\right) /\left(1+P_{t}\right)$.

We have already solved for the equity price process above. Using the fundamental pricing equation (4.1), we can solve for $P_{t}^{d}$ using the recursive relationship

$$
P_{t}^{d}=E_{t}\left(Q_{t, t+1}\left(1+P_{t+1}^{d}\right) \bar{g}\right)
$$

In our environment, (4.3) reduces to two equations in two unknowns. Using the components of the $Q$ process given by (4.2), and the transition matrix of (3.3) we obtain $P_{l}^{d}=45.984$ and $P_{h}^{d}=53.269$. The estimated upper bound on the welfare gains from eliminating consumption fluctuations completely vary with the current state, but not by much. I compute as upper bounds $\lambda_{l}=92 \%$ and $\lambda_{h}=88 \%$. So the welfare gains from eliminating all economic fluctuations, including the business cycle, are no more than about $90 \%$, but this is an enormous number!

Not all consumption fluctuations can be, nor should be, eliminated by discretionary policy. Can we compute an upper bound on the welfare gains from eliminating only those fluctuations that can and should be eliminated? I can think of two approaches.

In the first approach, I assume that stabilization policy can drive the economy back towards its trend path or "steer a tighter course". Policy makers often describe their goal as keeping the economy operating as close as possible to its potential. I formalize this by imagining a policy implemented at time $t$ that generates a consumption sequence of the form $\left\{\theta c_{t+s}+(1-\theta) \bar{g}^{s} c_{t}\right\}_{s=0}^{\infty}$. Notice that this new consumption sequence has the same mean as $\left\{c_{t+s}\right\}_{s=0}^{\infty}$; only the conditional variance will change. It is not hard to see that an upper bound, at least with homothetic preferences, on the welfare gains from moving to such a policy satisfies $\left(1+\lambda_{t}\right) \leq\left(\theta\left(1+P_{t}\right)+(1-\theta)\left(1+P_{t}^{d}\right)\right) /\left(1+P_{t}\right)$. Suppose we set $\theta=.86$, so that the conditional variance of one-year ahead consumption is reduced by about 30\%, a value loosely inspired by reading Lucas (2003). For this consumption path, I compute the upper bounds $\lambda_{l}=12.9 \%$ and $\lambda_{h}=12.3 \%$. Although substantially smaller than the bounds obtained for the policy that eliminates all consumption fluctuations, they leave plenty of room for substantive welfare gains.

A second approach, which mimics more closely the calculations and estimates in Alvarez and Jermann (2004), assumes that policy can generate 'smoothed' consumption sequences but cannot drive the economy back towards its trend path. This is formalized by imagining a policy implemented at time $t$ that generates a filtered consumption sequence of the form $\left\{\widetilde{c}_{t+s}\right\}_{s=0}^{\infty}$, where $\widetilde{c}_{t+s}=\theta_{0} c_{t+s}+\theta_{1} \bar{g} c_{t+s-1}+\ldots+\theta_{L} \bar{g}^{L} c_{t+s-L}$, where 
the weights are chosen to satisfy $\sum_{k=0}^{K} \theta_{k}=1$. Notice that the lagged consumption terms are scaled to have the same conditional mean as $c_{t+s}$. In general, the price of the filtered consumption sequence will be history dependent, so to simplify the calculations I follow Alvarez and Jermann (2004) and impose the "startup conditions" $\bar{g}^{k} c_{t-k}=c_{t}$. Because the pricing operator is no longer Markovian, pricing the filtered consumption sequence is a bit involved; details are presented in the appendix. But the intuition can be developed by considering the special case with $\widetilde{c}_{t+s}=\bar{g}^{L} c_{t+s-L}$. In the appendix, it is shown that claims to this sequence at time $t=0$ can be represented as the sum of two components: the deterministically growing coupon sequence $\left(1, \bar{g}, \ldots \bar{g}^{L-1}\right) c_{0}$ for $L-1$ periods, plus a claim to the payment stream of $\bar{g}^{L}$ units of the cum-dividend equity process, but received with a lag of $L$ periods. The value of this claim increases with $L$ : it ranges from $\left(1+P_{0}^{e}\right) c_{0}$ when $L=0$ to $\left(1+P_{0}^{d}\right) c_{0}$ as $L \rightarrow \infty$. The filtered consumption sequence has the same mean but it's uncertainty is postponed for $L$ periods and this can have an important effect on value. For example, the bound on the welfare gains from delivering $\left\{\widetilde{c}_{t+s}=\bar{g}^{L} c_{t+s-L}\right\}_{s=0}^{\infty}$ rather than $\left\{c_{t+s}\right\}_{s=0}^{\infty}$ is about $2 \%$ for $L=1$ and grows to about $17 \%$ at $L=10$.

Alvarez and Jermann (2004) choose the weights of the filtered consumption sequence to eliminate (as much as possible) the contribution of business cycle frequencies, that is cycles of eight years or less. The weights are reproduced for convenience in the appendix. Because the Alvarez and Jermann weights put $\theta_{0}=.625$ and assign almost no weight beyond two lags, it is not surprising the value of their filtered consumption sequence doesn't look very much different than that of the original sequence. I compute the upper bounds $\lambda_{l}=0.80 \%$ and $\lambda_{h}=0.78 \%$. Although my estimates of the welfare gains from eliminating business cycle frequency fluctuations in consumption are small, they are not quite as small as those reported in Alvarez and Jermann (2004). Part of the difference may be due to using the Mehra-Prescott economy as an approximation. However, I suspect that most of the difference comes from the fact that they calibrate to post-war quarterly data, whereas I use a longer sample of annual data. Nonetheless, we both agree that very little of the potential welfare gains from eliminating economic fluctuations would come from eliminating business cycle frequencies.

I conclude that the potential welfare gains from completing eliminating economic fluctuations may be very large. But the gains from eliminating high frequency variation in aggregate consumption are much more modest. The potential gains from better stabilization policy, if they can be achieved, will have to come from the reduction in medium and long-horizon uncertainty. 


\section{Estimating the Welfare Gains with Preferences}

The upper bounds on the welfare gains from completely eliminating consumption fluctuations that are obtained using asset prices are very large and a more informative estimate of the welfare gains may be obtained by using preference orderings. To obtain meaningful estimates, however, we must use preferences that are consistent with observed asset returns.

Standard CRRA preferences cannot generate the asset price process required to match the first two moments of equity and the riskfree rate in the Mehra-Prescott economy. With such preferences, it is well known that the predicted stochastic factors turn out to be of the form

$$
\widetilde{Q}_{i j}=\beta g_{j}^{-\gamma}
$$

where $\beta$ denotes the subjective discount rate and $\gamma$ is the coefficient of relative risk aversion. With CRRA preferences, the stochastic discount factor varies over time only with the rate of consumption growth realized next period. Comparing to (4.2), this says that the predicted two rows in the $Q$ matrix must be identical.

The Epstein and Zin (1989) preferences used by Tallarini (2000) lead to stochastic discount factors of the form

$$
\widetilde{Q}_{i j}=\beta g_{j}^{-\gamma}\left(\beta \frac{\left(1+P_{j}\right)}{P_{i}}\right)^{\delta}
$$

The dependence of this stochastic discount factor on $P_{i}$ removes the restriction that both rows of the predicted $Q$ matrix must be identical and replaces it with the requirement that it be of rank one (each row of $Q$ is a multiple of the first one). However, this is too restrictive to match (4.2) (which helps explain the failure of Epstein and Zin (2001) and Weil (1989) to find a significant improvement in matching the equity premium over results based on eq (5.1)), so the welfare gains assigned by these preferences to eliminating the business cycle must also be held in some doubt.

Melino and Yang (2003) generalize the preferences introduced by Epstein and Zin (1989) by allowing the parameters to be state dependent. This leads to a preference ordering over random consumption sequences that may be constructed by means of the following recursive functional relation:

$$
U_{t}=\left(c_{t}^{\rho\left(s_{t}\right)}+\beta\left(s_{t}\right)\left[E_{t}\left(U_{t+1}^{\alpha\left(s_{t}\right)}\right)\right]^{\frac{\rho\left(s_{t}\right)}{\alpha\left(s_{t}\right)}}\right)^{\frac{1}{\rho\left(s_{t}\right)}}, 0 \neq \alpha<1,0 \neq \rho<1 .
$$


where $E_{t}$ is the expectation conditional on period- $t$ information, $\beta\left(s_{t}\right), \alpha\left(s_{t}\right)$ and $\rho\left(s_{t}\right)$ are parameters that depend on an exogenous state variable $s_{t}$. For my purposes, I assume that $\beta\left(s_{t}\right)$ is a constant. The coefficient of relative risk aversion $(C R R A)$ for 'timeless gambles' implied by these preferences is $1-\alpha\left(s_{t}\right)$. The parameters $\beta$ and $\rho\left(s_{t}\right)$ are harder to interpret. When $\rho\left(s_{t}\right)=\rho$, a constant, $\beta$ is the discount parameter and measures time preference while the elasticity of intertemporal substitution $(E I S)$ is $1 /(1-\rho)$. For this reason, I will refer to $\beta$ as the discount parameter and $\rho\left(s_{t}\right)$ as the EIS parameter.

Melino and Yang (2003) show that with preferences given by (5.3), the intertemporal marginal rate substitution from time $t$ to $t+1\left(I M R S_{t, t+1}\right)$ is given by

$$
I M R S_{t, t+1}=\beta g_{t+1}^{\alpha\left(s_{t}\right)-1}\left(\frac{\beta M_{t, t+1}}{g_{t+1}}\right)^{\frac{\alpha\left(s_{t}\right)}{\rho\left(s_{t}\right)}-1}\left(a_{t+1}\right)^{\frac{\alpha\left(s_{t}\right)}{\rho\left(s_{t}\right)}-\frac{\alpha\left(s_{t}\right)}{\rho\left(s_{t+1}\right)}}
$$

where $M_{t, t+1}$ is the gross return to holding the optimal portfolio from time $t$ to $t+1$, and $a_{t} \equiv c_{t} / x_{t}$ is the consumption wealth ratio. Note that eq (5.4) exploits only information about the agent's preferences and her budget constraint. In a Lucas endowment economy, where a claim to the consumption stream is the only asset with positive net supply, the equilibrium conditions afford a further simplification.

If $\alpha\left(s_{t}\right)=\alpha$ (a constant) and $\rho\left(s_{t}\right)=\rho$ (a second constant), then eq(5.4) reduces to the stochastic discount factor implied by the model of Epstein and Zin (1989), namely

$$
I M R S_{t, t+1}^{E Z}=\beta g_{t+1}^{\alpha-1}\left(\frac{\beta M_{t, t+1}}{g_{t+1}}\right)^{\frac{\alpha}{\rho}-1}
$$

with $E I S=1 /(1-\rho)$ and $C R R A=1-\alpha$ (which equals the parameter $\gamma$ of $(5.2)$ ).

If $\alpha\left(s_{t}\right)=\rho\left(s_{t}\right)=\alpha$ (the same constant), eq(5.4) reduces further to the stochastic discount factor of CRRA preferences, namely.

$$
I M R S_{t, t+1}^{e u}=\beta g_{t+1}^{\alpha-1}
$$

with $E I S=1 /(1-\alpha)$ and $C R R A=1-\alpha$ (which equals the parameter $\gamma$ of (5.1)).

I consider only equilibria where the ex-dividend price of equity is described by the time-invariant and positive function, $p\left(g_{t}, c_{t}\right)$ of the variables $g_{t}$ and $c_{t}$. It follows, because of the homogeneity of preferences, that the price is linearly homogeneous in consumption; that is, 


$$
p(g, c)=p(g, 1) c=P(g) c .
$$

Thus the return to equity for the endowment economy simplifies to

$$
M_{t, t+1}=\frac{p\left(g_{t+1}, c_{t+1}\right)+c_{t+1}}{p\left(g_{t}, c_{t}\right)}=\frac{P\left(g_{t+1}\right)+1}{P\left(g_{t}\right)} g_{t+1} .
$$

For convenience, I use $P_{j}$ and $r_{j}$ to denote the price-dividend ratio and risk-free rate that obtain when the realized state is $g_{j}$.

In the Lucas endowment equilibrium, Melino and Yang (2003) show that the representative agent's wealth reduces to

$$
x_{t}=\left(c_{t}+p_{t}\right) \cdot 1
$$

so the consumption-wealth ratio in equilibrium can be written as

$$
a_{t} \equiv \frac{c_{t}}{x_{t}}=\frac{1}{1+p_{t} / c_{t}}=\frac{1}{1+P_{t}}
$$

where $P_{t} \equiv p_{t} / c_{t}$ is equity price-earnings ratio. Using eq(5.8) to replace $M_{t, t+1}$, and eq(5.10) to replace $a_{t+1}$ with $1 /\left(1+P_{t+1}\right)$, we see that in the equilibrium for this economy, the representative agent's intertemporal marginal rate substitution ${ }^{4}$ from time $t$ to $t+1$, given by eq(5.4), simplifies to give us a stochastic discount factor for this economy of the form

$$
Q_{t, t+1}=\beta g_{t+1}^{\alpha\left(s_{t}\right)-1}\left(P_{t} / \beta\right)^{1-\frac{\alpha\left(s_{t}\right)}{\rho\left(s_{t}\right)}}\left(1+P_{t+1}\right)^{\frac{\alpha\left(s_{t}\right)}{\rho\left(s_{t+1}\right)}-1}
$$

Notice that $Q_{t, t+1}$ varies explicitly with the current period's coefficient of relative risk aversion, $\alpha\left(s_{t}\right)$, but only implicitly on $\alpha\left(s_{t+1}\right)$ through the endogenous price-earnings process. By contrast, $Q_{t, t+1}$ varies explicitly with both current and next period's elasticity of intertemporal substitution, indexed by $\rho\left(s_{t}\right)$ and $\rho\left(s_{t+1}\right)$. Melino and Yang (2003) show that there exist many combinations of the parameter values in (5.11) that allow us to match the data given by (4.2).

In order to calculate the welfare gains with the Melino and Yang (2003) preferences, it is convenient to rewrite the preference ordering (5.3) as

$$
U_{t}=V_{t} c_{t}
$$

\footnotetext{
${ }^{4}$ I use the term 'stochastic discount factor' to describe the $I M R S$ after substituting terms involving market equilibrium.
} 
where $V_{t}$ satisfies the recursive relationship

$$
V_{t}=\left(1+\beta\left[E_{t}\left(V_{t+1}^{\alpha\left(s_{t}\right)} g_{t+1}^{\alpha\left(s_{t}\right)}\right)\right]^{\frac{\rho\left(s_{t}\right)}{\alpha\left(s_{t}\right)}}\right)^{\frac{1}{\rho\left(s_{t}\right)}}
$$

Note that $V_{t}$ measures the average utility per unit of consumption. With a slight abuse of notation, let $V_{0}$ denote the value that obtains when consumption growth is always constant and equal to its mean, i.e. $g_{t+1}=\bar{g}=1.018$. Using (5.13), we see that in the absence of uncertainty $V_{0}$ does not depend on the CRRA parameter, that is

$$
\begin{aligned}
V_{0}^{\rho(\bar{g})} & =\left(1+\beta\left[V_{0} \bar{g}\right]^{\rho(\bar{g})}\right) \\
& =\frac{1}{1-\beta \bar{g}^{\rho(\bar{g})}}
\end{aligned}
$$

where $\rho(\bar{g})$ denotes the EIS parameter of preferences that would obtain in a world of perfect certainty. Notice that (5.14) assigns exactly the same utility per unit of consumption as the CRRA preferences used by Lucas (2003), or the Epstein-Zin preferences used by Tallarini, for suitable choices of the parameter values. Ironically, there appears to be little disagreement about how to value deterministic consumption sequences, which is something that occurs only under the counterfactual. The controversy is about how to rank the random consumption sequences we see. It is important to point out that the preferences introduced by Melino and Yang (2003) are unusual, but they have the merit of matching the data whereas the other choices do not.

To compute the welfare gains from eliminating the business cycle, I compute the value of $\lambda$ that solves

$$
V_{t}\left(1+\lambda_{t}\right) c_{t}=V_{0} c_{t}
$$

The first five columns of Table 1, reproduced from Melino and Yang (2003) gives various combinations for the preference parameters so that (5.11) matches the data given by (4.2). Because the answers are relatively insensitive to which row of Table 1 we use for the preference parameters, I will focus on those based on the row with $\beta=.98$. I use linear interpolation to estimate the second preference parameter that appears in $V_{0}$, i.e. I set $\rho(\bar{g})=-2.04$, and compute $V_{0}=.2413$. In the high and low consumption growth states, I compute $V_{h}=.2010$ and $V_{l}=.1990$, respectively. So the welfare gains in the high and low consumption growth states to moving to a constant growth consumption sequence are $\lambda_{h}=20.1 \%$ and $\lambda_{l}=21.3 \%$, respectively. In contrast, if we use the CRRA preferences of Lucas with $\gamma=1-\rho(\bar{g}) \approx 3$, we would estimate $\lambda=0.25 \%$.

We can get different estimates by using other rows from Table 1, but the basic result is extremely robust. If we have preferences that match the data on asset returns, then 
the welfare gains from eliminating economic fluctuations completely are two orders of magnitude larger than those reported by Lucas (2003). While there is no reason to expect future research to achieve a complete elimination of the business cycle, nor can we be sure that such research would necessarily pass a cost-benefit analysis, we should place very little confidence in the Lucas' estimate of the upper bound for possible gains.

\section{Conclusion}

Lucas (2003) argues that the welfare gains from further reductions in business cycle fluctuations are small and that macroeconomists should adjust their priorities and work on problems, such as improving the long run performance of the economy, that promise larger returns. I show that Lucas' procedure, when calibrated to the last century of data rather than just to the post-war period, yields the troubling conclusion that macroeconomists should never have worried about the business cycle in the first place. I argue that the problem with Lucas' calculations comes from using preferences that are inconsistent with observed asset return data. The preferences introduced by Melino and Yang (2003) agree with those used by Lucas for valuing consumption sequences that grow at a constant rate. However, unlike the CRRA preferences used by Lucas, the Melino and Yang preferences also match the historical data on the first two moments of the returns on the risk-free bond and equity. Using preferences that are consistent with observed asset prices raises Lucas' welfare estimates from elimination of economic fluctuations completely by two orders of magnitude and makes it as large as other feasible policies that he lists.

The gains from further reductions in economic fluctuations, however, are estimated to depend crucially on which frequencies are attenuated. There appears to be very little to gain from smoothing out year to year fluctuations. Asset markets reveal that the large potential gains in welfare would come if we could steer a tighter course and reduce the medium and long-horizon uncertainty about the path of aggregate consumption. Whether or not policies can be found to achieve such a reduction deserves further research. 
Figure 1: Upper Bound on Welfare Gains with Homothetic Preferences

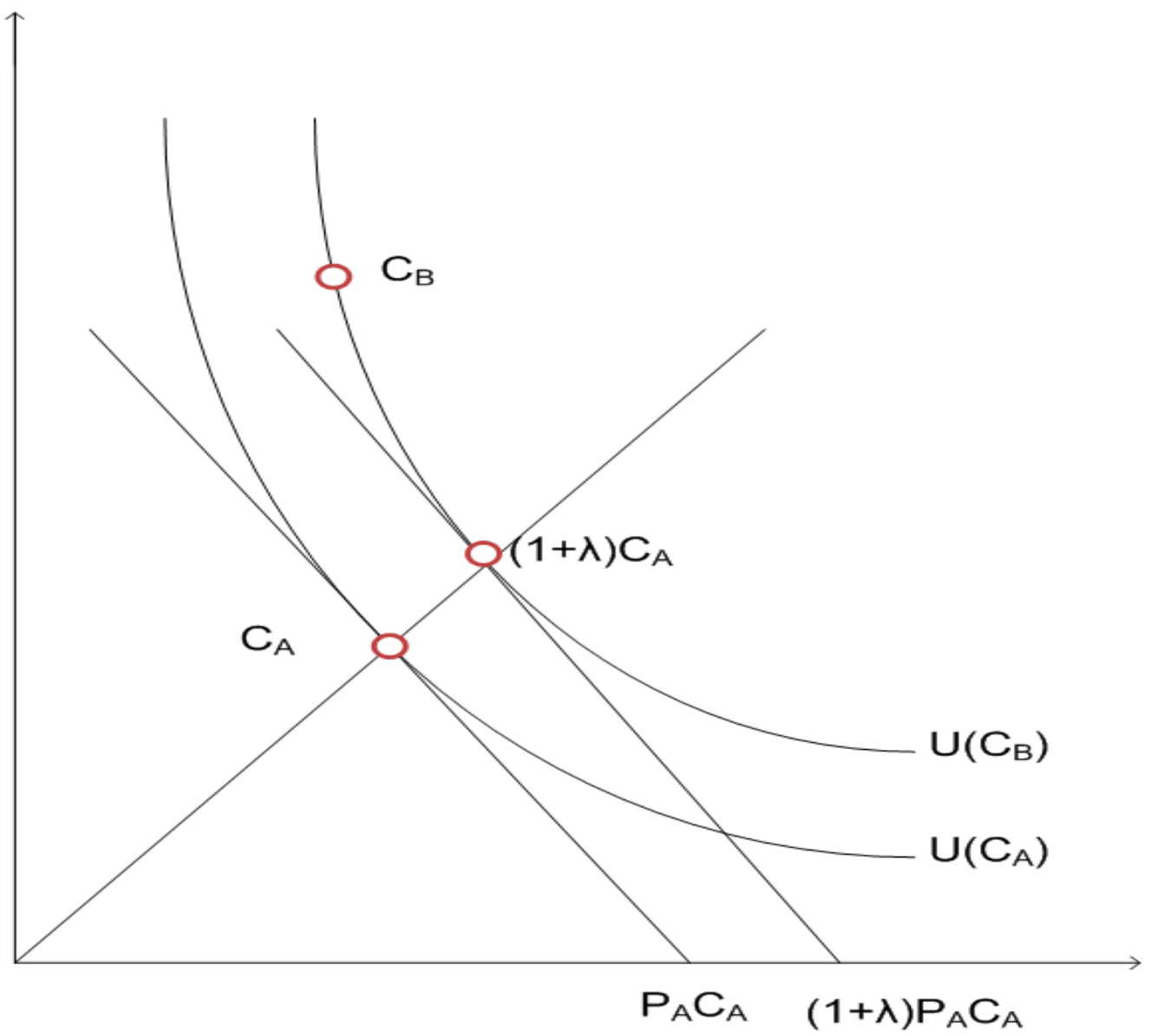


Table 1: Welfare Gains from Eliminating Business Cycles Completely

\begin{tabular}{rcccccccccc}
\hline \hline$\beta$ & $\alpha_{l}$ & $\alpha_{h}$ & $\rho_{l}$ & $\rho_{h}$ & $\rho(\bar{g})$ & $V_{0}$ & $V_{l}$ & $V_{h}$ & $\lambda_{l}$ & $\lambda_{h}$ \\
.97 & -21.21 & .76 & -.92 & -.97 & -.945 & .0386 & .0309 & .0314 & $25.0 \%$ & $23.2 \%$ \\
.98 & -22.25 & .79 & -1.98 & -2.10 & -2.04 & .2413 & .1990 & .2010 & $21.3 \%$ & $20.1 \%$ \\
.99 & -22.57 & .81 & -3.04 & -3.22 & -3.13 & .4150 & .3493 & .3524 & $17.8 \%$ & $18.8 \%$ \\
\hline
\end{tabular}

The first five columns are taken from Melino and Yang (2003), Table 4. For each value of $\beta$, the preference parameters $\alpha_{l}, \alpha_{h}, \rho_{l}$ and $\rho_{h}$ are chosen to price the required equity and risk free rate processes exactly. The deterministic, constantly growing consumption sequence is evaluated using the given value of $\beta$ and the elasticity of intertemporal substitution parameter $\rho(\bar{g})$. $V_{0} c_{t}, V_{l} c_{t}$, and $V_{h} c_{t}$ give the the utility levels for the deterministic consumption sequence, and the Mehra-Prescott endowment sequence in the low growth and high growth states, respectively. The welfare gain from moving to the deterministic, constantly growing consumption sequence is given by the value of $\lambda_{l}$ and $\lambda_{h}$. 


\section{References}

[1] Alvarez, F. and U.J. Jermann, (2004), 'Using asset prices to measure the cost of business cycles', Journal of Political Economy, 112(6), p1223-1256.

[2] Epstein, L.G. and A. Melino, (1995), 'A revealed preference analysis of asset pricing under recursive utility', Review of Economic Studies, 62(4), p597-618.

[3] Epstein, L.G. and S.E. Zin, (1989), 'Substitution, risk aversion and the temporal behavior of consumption and asset returns: A theoretical framework.', Econometrica, 57(4), p937-969.

[4] Epstein, L.G. and S.E. Zin, (1990), "First-order' risk aversion and the equity premium puzzle', Journal of Monetary Economics, 26(3), p387-407.

[5] Epstein, L.G. and S.E. Zin, (2001), 'The Independence Axiom and asset returns', Journal of Empirical Finance, 8(5), p537-572.

[6] Hansen, L.P. and R. Jagannathan, (1991) 'Implications of Security Market Data for Models of Dynamic Economies', Journal of Political Economy, 99(2), p225-262.

[7] Hansen, L.P. and S. F. Richard (1987), 'The role of conditioning information in deducing testable restrictions implied by dynamic asset pricing models,' Econometrica, 55(3), 587-613.

[8] Lucas, R.E., (2003), 'Macroeconomic Priorities', American Economic Review, 93(1), p1-14

[9] Mehra, R. and E.C. Prescott, (1985), 'The equity premium: a puzzle', Journal of Monetary Economics, 15(2), p145-161.

[10] Melino, A. and A. Yang (2003) 'State-dependent preferences can explain the equity premium puzzle' Review of Economic Dynamics 6(4), p806-830.

[11] Prescott, E.C. (2002) 'Richard T. Ely Lecture: Prosperity and Depression', American Economic Review, 92(2), p1-15

[12] Tallarini, T.D., Jr. (2000), 'Risk-sensitive real business cycles', Journal of Monetary Economics, 45(3), p507-532.

[13] Weil, P., (1989), 'The equity premium puzzle and the risk-free rate puzzle', Journal of Monetary Economics, 24(3), p401-421.

[14] Yang, Alan X. (2001), An investigation of asset pricing puzzles with cyclical risk aversion and intertemporal substitution, dissertation, University of Toronto 


\section{Appendix}

This appendix shows how to compute the value a filtered consumption sequence: $\widetilde{c}_{t}=\theta_{0} c_{t}+\theta_{1} \bar{g} c_{t-1}+\ldots+\theta_{L} \bar{g}^{L} c_{t-L}$

Let $Q_{0, t}$ denote the stochastic discount process that gives the time 0 price for payoffs at time $t$. Using

i) Law of Iterated Expectations

ii) $Q_{0, t}=Q_{0, t-1} Q_{t-1, t}$

iii) $c_{t}=g_{t} c_{t-1}$

we have

$$
\begin{aligned}
E_{0}\left(Q_{0, t} c_{t}\right) & \left.=E_{0}\left(E_{1} Q_{0, t} c_{t}\right)\right) \\
& =E_{0}\left(Q_{0,1} g_{1} E_{1}\left(Q_{1, t} g_{2} \cdots g_{t}\right)\right) c_{0}
\end{aligned}
$$

Define the matrices

$$
R=\left[\begin{array}{cc}
\pi_{l l} Q_{l l} & \pi_{l h} Q_{l h} \\
\pi_{h l} Q_{h l} & \pi_{h h} Q_{h h}
\end{array}\right] \quad, \quad D_{g}=\left[\begin{array}{cc}
g_{l} & 0 \\
0 & g_{h}
\end{array}\right]
$$

and the vectors

$$
\mathbb{E}_{t}(X)=\left[\begin{array}{c}
E\left(X \mid g_{t}=g_{l}\right) \\
E\left(X \mid g_{t}=g_{h}\right)
\end{array}\right], \quad \iota=\left[\begin{array}{l}
1 \\
1
\end{array}\right]
$$

The current price of one future unit of consumption at time t can be written as

$$
\begin{aligned}
\mathbb{E}_{0}\left(Q_{0, t}\right) & =\left[\begin{array}{c}
\pi_{l l} Q_{l l} g E\left(Q_{1, t} \mid g_{1}=g_{l}\right)+\pi_{l h} Q_{l h} E\left(Q_{1, t} \mid g_{1}=g_{h}\right) \\
\pi_{h l} Q_{h l} E\left(Q_{1, t} \mid g_{1}=g_{l}\right)+\pi_{h h} Q_{h h} E\left(Q_{1, t} \mid g_{1}=g_{h}\right)
\end{array}\right] \\
& =\left[\begin{array}{cc}
\pi_{l l} Q_{l l} & \pi_{l h} Q_{l h} \\
\pi_{h l} Q_{h l} & \pi_{h h} Q_{h h}
\end{array}\right] \mathbb{E}_{1}\left(Q_{1, t}\right)=R \mathbb{E}_{1}\left(Q_{1, t}\right) \\
& =R^{t} \iota
\end{aligned}
$$

Similarly, we can write

$$
\begin{aligned}
\mathbb{E}_{0}\left(Q_{0, t} \frac{c_{t}}{c_{0}}\right) & =\left[\begin{array}{c}
\pi_{l l} Q_{l l} g_{l} E\left(Q_{1, t} g_{2} \cdots g_{t} \mid g_{1}=g_{l}\right)+\pi_{l h} Q_{l h} g_{h} E\left(Q_{1, t} g_{2} \cdots g_{t} \mid g_{1}=g_{h}\right) \\
\pi_{h l} Q_{h l} g_{l} E\left(Q_{1, t} g_{2} \cdots g_{t} \mid g_{1}=g_{l}\right)+\pi_{h h} Q_{h h} g_{h} E\left(Q_{1, t} g_{2} \cdots g_{t} \mid g_{1}=g_{h}\right)
\end{array}\right] \\
& =\left[\begin{array}{cc}
\pi_{l l} Q_{l l} g_{l} & \pi_{l h} Q_{l h} g_{h} \\
\pi_{h l} Q_{h l} g_{l} & \pi_{h h} Q_{h h} g_{h}
\end{array}\right] \mathbb{E}_{1}\left(Q_{1, t} g_{2} \cdots g_{t}\right)=\left(R D_{g}\right) \mathbb{E}_{1}\left(Q_{1, t} g_{2} \cdots g_{t}\right) \\
& =\left(R D_{g}\right)^{t} \iota
\end{aligned}
$$

Combining the two results above, we have for $0 \leq \tau \leq t$

$$
\mathbb{E}_{0}\left(Q_{0, t} \frac{c_{\tau}}{c_{0}}\right)=\mathbb{E}_{0}\left(Q_{0, \tau} \frac{c_{\tau}}{c_{0}} E_{\tau} Q_{\tau, t}\right)=\left(R D_{g}\right)^{\tau} R^{t-\tau} \iota
$$


As an application, let's compute the cum-dividend price of the equity process. Using obvious notation,

$$
\begin{aligned}
\iota+\mathbb{P}^{e} & =\mathbb{E}_{0}\left(\sum_{t=0}^{\infty} Q_{0, t} \frac{c_{t}}{c_{0}}\right) \\
& =\sum_{t=0}^{\infty}\left(R D_{g}\right)^{t} \iota=\left(I-R D_{g}\right)^{-1} \iota
\end{aligned}
$$

Similary, the price of the geometrically-growing coupon bond process satisfies

$$
\begin{aligned}
\iota+\mathbb{P}^{d} & =\mathbb{E}_{0}\left(\sum_{t=0}^{\infty} Q_{0, t} \frac{\bar{g}^{t} c_{0}}{c_{0}}\right) \\
& =\sum_{t=0}^{\infty} R^{t} \bar{g}^{t} \iota \\
& =(I-R \bar{g})^{-1} \iota
\end{aligned}
$$

Plugging in the values from the text,

$$
\Pi=\left[\begin{array}{ll}
.43 & .57 \\
.57 & .43
\end{array}\right], \quad D_{g}=\left[\begin{array}{cc}
0.982 & 0 \\
0 & 1.054
\end{array}\right], \quad \bar{g}=1.018
$$

and carrying extra digits in $Q$ to reduce rounding errors, namely,

$$
Q=\left[\begin{array}{ll}
1.8623244 & 0.2439476 \\
1.1265597 & 0.9494910
\end{array}\right]
$$

we obtain

$$
R=\left[\begin{array}{ll}
.800799492 & .139050132 \\
.642139029 & .408281130
\end{array}\right]
$$

and

$$
\mathbb{P}^{e}=\left[\begin{array}{l}
23.467 \\
27.838
\end{array}\right] \quad \text { and } \quad \mathbb{P}^{d}=\left[\begin{array}{l}
45.984 \\
53.269
\end{array}\right]
$$

Note that the largest eigenvalue of $R$ is .962 , so the infinite sums above all converge.

Now consider the filtered consumption process

$$
\widetilde{c}_{t}=\theta_{0} c_{t}+\theta_{1} \bar{g} c_{t-1}+\ldots+\theta_{L} \bar{g}^{L} c_{t-L}=\sum_{i=0}^{L} \theta_{i} \bar{g}^{i} c_{t-i}
$$


with startup conditions: $c_{0}=\bar{g}^{s} c_{-s}$ for $s=1 \ldots L$. Assume the weights sum to one, i.e. $\sum_{i=0}^{L} \theta_{i}=1$, which implies $\widetilde{c}_{0}=c_{0}$. The cum-dividend price of the asset that pays off the filtered consumption sequence satisfies

$$
\left(\iota+\widetilde{\mathbb{P}}^{e}\right) c_{0}=\mathbb{E}_{0}\left(\sum_{t=0}^{\infty} Q_{0, t} \widetilde{c}_{t}\right)
$$

Therefore,

$$
\begin{aligned}
\iota+\widetilde{\mathbb{P}}^{e} & =\mathbb{E}_{0}\left(\sum_{t=0}^{\infty} Q_{0, t} \frac{\widetilde{c}_{t}}{c_{0}}\right) \\
& =\mathbb{E}_{0}\left(\sum_{t=0}^{\infty} Q_{0, t} \frac{\sum_{i=0}^{L} \theta_{i} \bar{g}^{i} c_{t-i}}{c_{0}}\right) \\
& =\sum_{i=0}^{L} \theta_{i} \bar{g}^{i} \mathbb{E}_{0}\left(\sum_{t=0}^{\infty} Q_{0, t} \frac{c_{t-i}}{c_{0}}\right) \\
& =\sum_{i=0}^{L} \theta_{i} \bar{g}^{i}\left[\sum_{t=0}^{i-1} \mathbb{E}_{0}\left(Q_{0, t}\right) \frac{c_{t-i}}{c_{0}}+\sum_{t=i}^{\infty} \mathbb{E}_{0}\left(Q_{0, t} \frac{c_{t-i}}{c_{0}}\right)\right]
\end{aligned}
$$

Rewriting the two sums slightly (put $j=i-t$ in first sum and $s=t-i$ in second sum) and assumping that consumption before time $t=0$ satisfies $c_{0}=\bar{g}^{j} c_{-j}$, we obtain

$$
\begin{aligned}
\iota+\widetilde{\mathbb{P}}^{e} & =\sum_{i=0}^{L} \theta_{i} \bar{g}^{i}\left[\sum_{j=1}^{i} \mathbb{E}_{0}\left(Q_{0, i-j}\right) \frac{c_{-j}}{c_{0}}+\sum_{s=0}^{\infty} \mathbb{E}_{0}\left(Q_{0, s+i} \frac{c_{s}}{c_{0}}\right)\right] \\
& =\sum_{i=0}^{L} \theta_{i}\left[\sum_{j=1}^{i} \mathbb{E}_{0}\left(Q_{0, i-j}\right) \bar{g}^{i-j}+\bar{g}^{i} \sum_{s=0}^{\infty} \mathbb{E}_{0}\left(Q_{0, s+i} \frac{c_{s}}{c_{0}}\right)\right] \\
& =\sum_{i=0}^{L} \theta_{i}\left[\sum_{j=0}^{i-1} R^{j} \bar{g}^{j}+\bar{g}^{i} \sum_{s=0}^{\infty}\left(R D_{g}\right)^{s} R^{i}\right] \iota
\end{aligned}
$$

The first term in square brackets gives the value of the geometrically-growing coupon sequence up to time $i-1$; the second term gives the value of the claim to $\bar{g}^{i}$ units of 
the cum-dividend equity payoffs, but received with a lag of $i$ periods. We can rewrite this expression slightly in terms of $\mathbb{P}^{e}$ and $\mathbb{P}^{B}$,

$$
\begin{aligned}
\iota+\widetilde{\mathbb{P}}^{e} & =\sum_{i=0}^{L} \theta_{i}\left[\left(I-R^{i} \bar{g}^{i}\right)(I-R \bar{g})^{-1}+\left(I-R D_{g}\right)^{-1} R^{i} \bar{g}^{i}\right] \iota \\
& =\sum_{i=0}^{L} \theta_{i}\left[\left(I-R^{i} \bar{g}^{i}\right)\left(\iota+\mathbb{P}^{d}\right)+\left(I-R D_{g}\right)^{-1} R^{i} \bar{g}^{i}\left(I-R D_{g}\right)\left(\iota+\mathbb{P}^{e}\right)\right] \\
& =\iota+\mathbb{P}^{e}+\sum_{i=0}^{L} \theta_{i}\left[\left(I-R^{i} \bar{g}^{i}\right)\left(\iota+\mathbb{P}^{d}\right)-\left(I-R D_{g}\right)^{-1}\left(I-R^{i} \bar{g}^{i}\right)\left(I-R D_{g}\right)\left(\iota+\mathbb{P}^{e}\right)\right]
\end{aligned}
$$

Clearly, the value of the filtered consumption process depends on the weights $\theta_{i}$ and in a complicated way on the discount matrix $R$. If we use the spectral decomposition of $R$, we can write for our data

$$
R^{i}=(.962)^{i}\left(\begin{array}{l}
.653 \\
.757
\end{array}\right)\left(\begin{array}{ll}
1.186 & .298
\end{array}\right)+(.247)^{i}\left(\begin{array}{c}
-.298 \\
1.186
\end{array}\right)\left(\begin{array}{ll}
-.757 & .653
\end{array}\right)
$$

So the price of the filtered consumption process is given by

$$
1+\widetilde{\mathbb{P}}^{e} \approx\left[\begin{array}{l}
46.984 \\
54.269
\end{array}\right]-\sum_{i=0}^{L} \theta_{i}(1.018)^{i}\left((.962)^{i}\left[\begin{array}{l}
22.387 \\
25.952
\end{array}\right]+(.247)^{i}\left[\begin{array}{l}
.056 \\
.068
\end{array}\right]\right)
$$

Note that the Alvarez and Jermann (2004, Appendix E) filter weights are

$$
\theta=\begin{array}{ccccccc}
{[0.6250} & 0.2251 & 0.1592 & 0.0750 & -0.0000 & -0.0450 & -0.0531 \\
-0.0322 & 0.0000 & 0.0250 & 0.0319 & 0.0205 & -0.0000 & -0.0173 \\
-0.0228 & -0.0150 & 0.0000 & 0.0133 & 0.0177 & 0.0119 & -0.0191]
\end{array}
$$

